Portland State University

PDXScholar

\title{
A psychometric evaluation of the Bicycle drawing test and the establishment of preliminary norms
}

\author{
Mary Lee Nichols \\ Portland State University
}

Follow this and additional works at: https://pdxscholar.library.pdx.edu/open_access_etds

Part of the Cognition and Perception Commons

Let us know how access to this document benefits you.

\section{Recommended Citation}

Nichols, Mary Lee, "A psychometric evaluation of the Bicycle drawing test and the establishment of preliminary norms" (1980). Dissertations and Theses. Paper 3075.

https://doi.org/10.15760/etd.3069

This Thesis is brought to you for free and open access. It has been accepted for inclusion in Dissertations and Theses by an authorized administrator of PDXScholar. Please contact us if we can make this document more accessible: pdxscholar@pdx.edu. 
AN ABSTRACT OF THE THESIS OF Mary Lee Nichols for the Master of Science in Psychology presented December 2, 1980.

Title: A Psychometric Evaluation of the Bicycle Drawing Test and the Establishment of Preliminary Norms

APPROVED BY MEMBERS OF THE THESIS COMMITTEE:

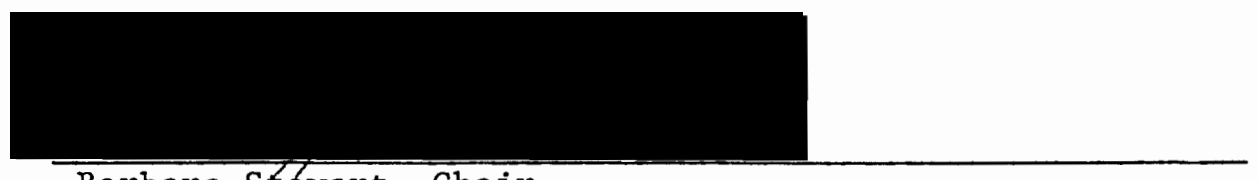

Barbara Stewart, Chair

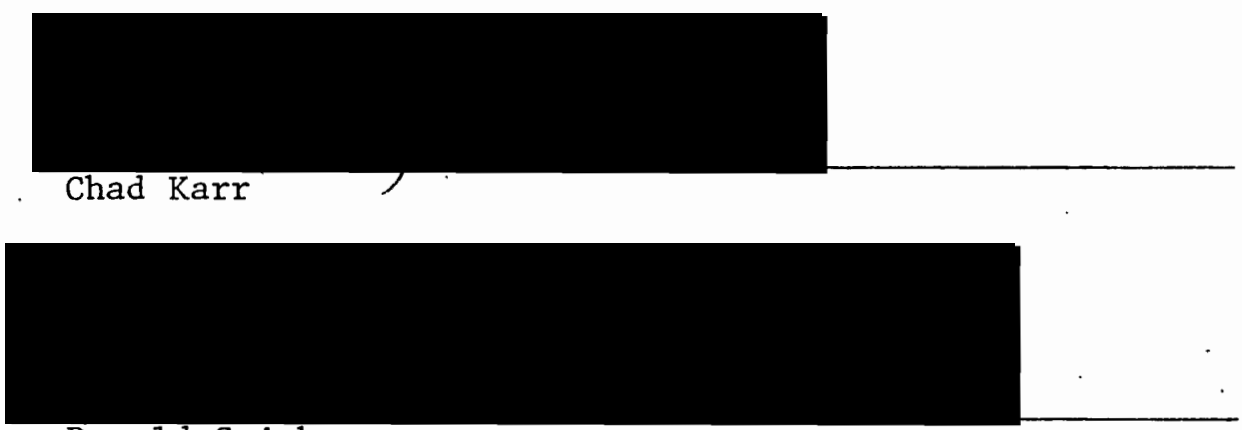

Ronald Smith

The main purpose of this study was to examine the psychometric characteristics of the Bicycle Drawing Test. The 20-point scoring criterion was evaluated, and the preliminary norms were established.

The Bicycle Drawing Test is an easily administered free-style drawing task that has been found to be a useful addition to a neuropsychological test battery. It provides a sample of visuopractic functioning involving the formation of a perceptual construct, a motor response, and a spatial component. 
One hundred-forty-one adult male volunteers who were patients at a disability evaluation center for injured workers participated in the study. Their ages ranged from 20 to 64 years. Five age categories were used to determine the effect of age on drawing performance.

The Bicycle Drawing Test was administered to all participants who had also taken the Wechsler Adult Intelligence Scale (WAIS) and the General Aptitude Test Battery (GATB). Hypotheses were made regarding correlations of the Bicycle Drawing Test with subtests of the WAIS and GATB thought to measure similar abilities. As hypothesized, Block Design and Object Assembly subtests of the WAIS had high correlations with the Bicycle Drawing Test. The high correlations with Picture Completion, Similarities, and Information had not been predicted. Hypothesized correlations of the Bicycle Drawing Test with GATB subtests Three-Dimensional Space and Form Matching were found and offer evidence for the validity of the Bicycle Drawing Test.

Internal consistency reliability and interscorer reliability meet standards for tests of this sort. Test-retest reliability is not as high as desired. Possible reasons for this have been offered and suggestions have been made for possible remedies.

Handedness and age were examined as variables. Neither was found to have a significant effect on performance on this test.

The study presents some normative data on the Bicycle Drawing Test. The data indicate that this test, while it should not be used alone to draw conclusions about presence of organicity, has an appropriate place in a neuropsychological test battery. 
A PSYCHOMETRIC EVALUATION OF THE BICYCLE DRAWING TEST AND THE ESTABLISHMENT OF PRELIMINARY NORMS

by

MARY LEE NICHOLS

A thesis submitted in partial fulfillment of the requirements for the degree of

\author{
MASTER OF SCIENCE \\ in \\ PSYCHOLOGY
}

Portland State University 
TO THE OFFICE OF GRADUATE STUDIES AND RESEARCH:

The members of the Committee approve the thesis of Mary Lee Nichols presented December 2, 1980.

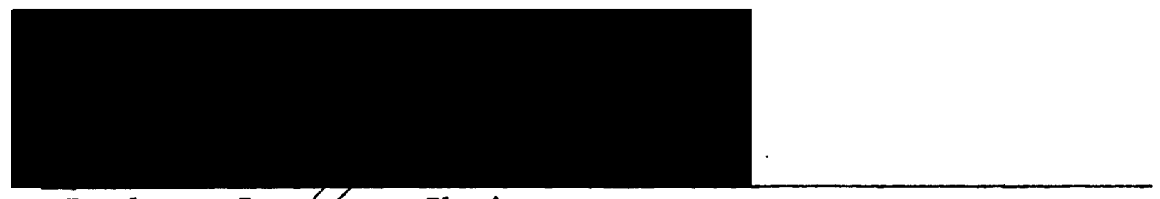

Barbara Stefart, Chair

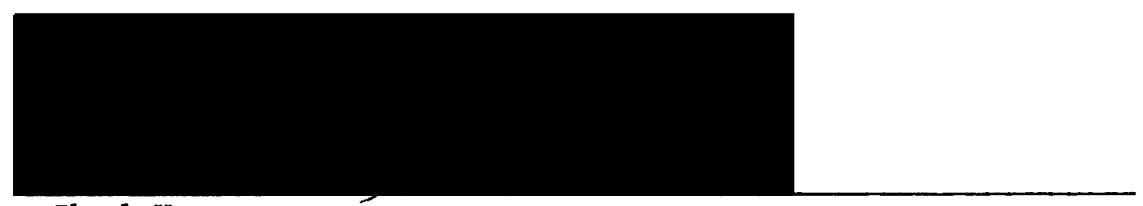

Chad Karr

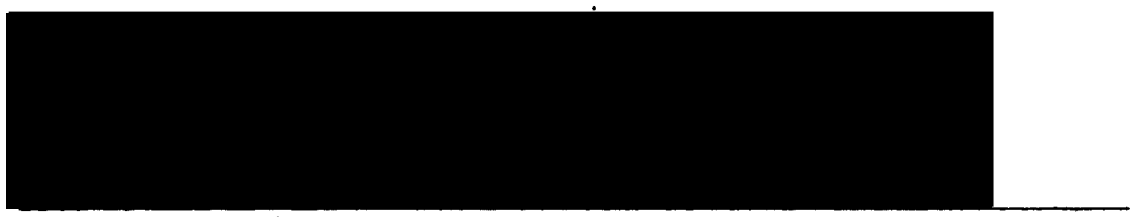

Ronald Smith

APPROVED :

Robert Jones, Head, Department of Psychology

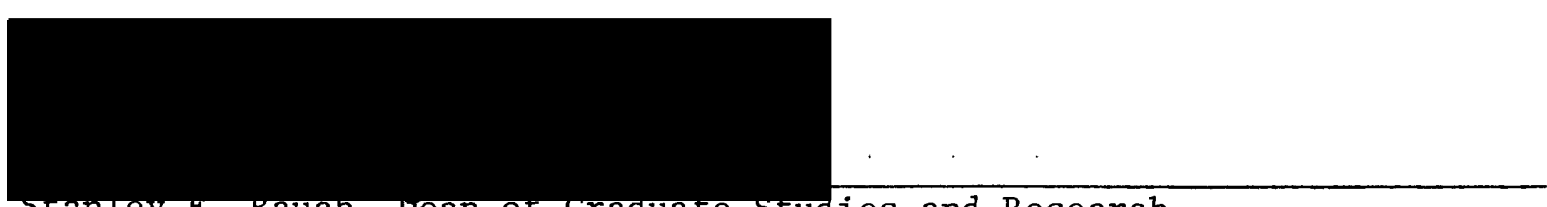

stanley E. Kaucn, vean of Graduate studies and Research 


\section{ACKNOWLEDGEMENTS}

I would like to express my gratitude to all the people who helped me in this learning experience. Muriel Lezak suggested the topic and was very helpful in conceptualization and design of the project. Barbara Stewart gave generously of her time, providing support, encouragement, and expert guidance in all stages from planning through the writing of results. I would thank Chad Karr and Ronald Smith for serving on my committee, offering support, raising issues that needed consideration, and stimulating new ideas.

I am grateful to Rosemary McBrayer who typed the many drafts of this manuscript, to Judy Howard who assisted with the editing and typing, to Donna Stuteville who programmed the computer, to Helen Tuggy who scored the tests, and to my daughter, Donna, who helped code the data.

I would like to thank Worker's Compensation Board Disability Center, now called Callahan Center, in Wilsonville, Oregon, for cooperating in allowing me to collect data there and to all the volunteers who participated in the project. Psychometrists Judi Brown, Tom Danner, and Eva Kautz assisted me by screening participants and administering the tests. Their cooperation in the collection of data shortened the process by many months. I thank Paul Slattery for his help in researching the literature and editing the manuscript.

I owe special thanks to my family for their encouragement and support. My parents and siblings, especially Derald, maintained an interest in this project that ensured its completion. My greatest thanks go to my children 
for their continuous support, understanding and endurance during this venture and the long years of acquiring my education. 
TABLE OF CONTENTS

PAGE

ACKNOWLEDGEMENTS . • . . . . . . . . . . . • . • • • iii

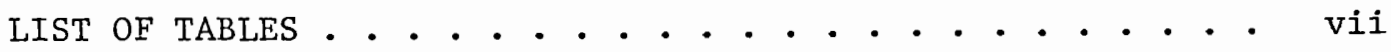

INTRODUCTION . . . . . . . . . . . . . . . . . . . . 1

Statement of Purpose of Study . . . . . . . . . . . 2

Review of Literature . . . . . . . . . . . . 2

METHOD . . . . . . . . . . . . . . . . . . 12

Subjects • . . . . . . . . . . . . . . . . 12

Instruments . . . . . . . . . . . . . . . 13

Wechsler Adult Intelligence Scale . . . . . . 13

General Aptitude Test Battery . . . . . . . 15

Bicycle Drawing Test . . . . . . . . . . 19

Procedure . . . . . . . . . . . . . . . . 20

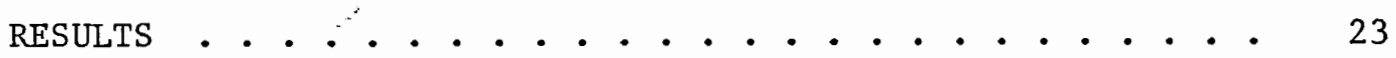

Reliability of Bicycle Drawing Test . . . . . . . . 23

Interscorer Reliability . . . . . . . . . 23

Test-Retest Reliability . . . . . . . . 25

Internal Consistency Reliability . . . . . . 25

Validity of Bicycle Drawing Test . . . . . . . . 28

Other Evaluations of Bicycle Drawing Test . . . . . . 30

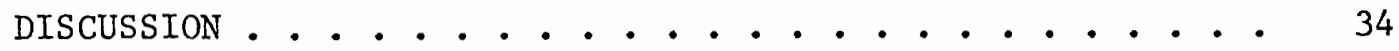

REFERENCES . . . . . . . . . . . . . . . . . . . 40 
PAGE

\section{APPENDICES}

Table of Normative Data for Subtests of WAIS . . . . . 43

Means and Standard Deviations for Subtests of WAIS for Study Sample. . . . . . . . . . . 44

Scoring: Bicycle Drawing Test . . . . . . . . . 45

Bicycle Drawing Test Questionnaire . . . . . . . . 46 


\section{LIST OF TABLES}

TABLE

PAGE

1 Interscorer Reliability on Bicycle Drawing

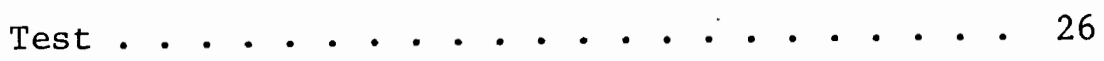

2 Test-Retest Reliability . . . . . . . . . . 27

3 Item Difficulty and Corrected Item - Total

Correlations for the 20 Bicycle Drawing Test

Items . . . . . . . . . . . . 29

4 Correlation of Bicycle Drawing Test with WAIS

Subtests . . . . . . . . . . . . 31

5 Correlation of Bicycle Drawing Test with GATB

Subtests. . . . . . . . . . . . . 32

6 Means and Standard Deviations of Bicycle Drawing

Test Total Scores . . . . . . . . . . 33 
A PSYCHOMETRIC EVALUATION OF THE BICYCLE DRAWING TEST AND THE ESTABLISHMENT OF PRELIMINARY NORMS

The Bicycle Drawing Test is an easily administered test that can be useful in eliciting visuopractic or visuoconceptual dysfunctions or aberrations in the handling of space. The test has the advantages of requiring only pencil, paper, and simple instructions, being quick to administer, and being applicable to all but persons so severely handicapped that they cannot draw (Lezak, 1976). The test was adapted from Piaget (1930) who developed it as a procedure to study children's reasoning. Taylor (1959) suggested the drawing served only as a concrete basis for discussion by which to appraise the child's comprehension and the quality of his thought process. The test has since been found to be a useful addition to a neuropsychological test battery. As a free drawing test, it provides a sample of visuopractic functioning involving the formation of a perceptual construct, a motor response, and a spatial component (Lezak, 1976).

The Bicycle Drawing Test may demonstrate differential contribution of left and right hemispheres in production of a complete drawing. The right hemisphere appears to involve the ability to see the gestalt, the whole of an object, rather than seeing it as a collection of parts. Some patients with right hemisphere damage may remember and draw many components of the bicycle but without maintaining the overall proportions. Carefully drawn details may be misplaced in relation to one another. It is as if these patients think of the parts one at a time and draw them without concern for how they are spacing them. Sometimes the details are 
unnecessarily elaborate and extensive.

Patients with left hemisphere damage are more likely to preserve the overall proportions but to produce a greatly oversimplified machine (McFie \& Zangwill, 1960). Drawings by these patients may look primitive or childlike. Parts of the bicycle may be missing; for example, seat, wheels, chain, or pedals (Warrington, James, \& Kinsbourne, 1966).

The Bicycle Drawing Test can also serve as a test of mechanical reasoning. The experimenter who is interested in whether the subject can think through the sequential operation of the machine can ask, "How does it work?" (Taylor, 1959).

\section{Purpose of the Present Study}

The purpose of the present study is to examine the psychometric characteristics of the Bicycle Drawing Test, the method of scoring the test devised by Lezak, and to look at other cognitive variables which are related to performance on the test. Adult norms have not been established. The present study establishes preliminary norms.

Review of the Literature

There are numerous references in the literature to the use of bicycle drawings as a task that elicits demonstration of certain abilities, but few studies that make an attempt to score the results objectively. McFie (1975) points out that although the free-style drawing of a bicycle may be beyond the ability of some normal persons, it is well suited to illustrate the difference between types of impairment. Free-style drawing 
tests, as opposed to a copying task, require the arousal of a perceptual construct (Lezak, 1976). They also display the manner in which the person copes with the spatial elements of a composition. By adopting certain themes, such as a bicycle, as a standard test, an abnormal performance is readily recognized and the performance deficits can be analyzed. The bicycle is a particularly useful test theme because it entails the fitting together of a number of essential parts. Confusion of two and three dimensions can be easily identified. Being a "closed" object, unilateral neglect (performing as if one half of the object does not exist) is easily recognized (Critchley, 1953). It must be considered that some subjects may have had limited experience with bicycles; however, bicycles are found in most cultures (S1attery, 1980).

A search of the literature, including libraries and three bibliographic retrieval services (Psychology Abstracts, Excerpta Medica, and Medline) located only two studies that involved the use of evaluation of the bicycle drawing test as an instrument with objective measurements. One of the studies was conducted at Punjabi University in Patiala, India by T.R. Sharma. Sharma constructed and standardized a 75-point bicycle drawing scale for measuring intelligence of 11- to 16-year-old children. Age norms were established. No sex difference in intelligence score was found. Reliability coefficient of the test for the different age groups, using test-retest, split half, and $\mathrm{KR}-21$ were all above .80 . Validity coefficients were also determined by correlating test scores with teachers' estimate, children's scholastic achievement, the Goodenough "Draw A Man" Test, and Jenkin's Test (Sharma, 1972). 
The only study that evaluated drawing tests as neuropsychological assessment instruments was conducted by Warrington, James, and Kinsbourne (1966). As the bench mark study in this field, it provides a basis for comparison for subsequent similar studies, including the present one. Warrington et al. examined evidence for separate hemispheric contributions to the drawing process by quantifying the predominant error types in relation to laterality of lesion. They saw the necessity for having an objective scoring system rather than relying on the subjective assessment by experimenters. They also saw the need for experimental groups being matched for severity of drawing deficits and suggest that earlier positive results showing hemispheric differences may have been due to lack of control for differences in severity of disability. Warrington et al. cite a study by Arrigori and DeRenzi that did control for severity differences by matching groups on a visual reaction time, thought not to be sensitive to lesions of either hemisphere. They found no demonstrable difference in drawing disability in left and right hemisphere patients.

Warrington et al. further assert that misleading conclusions are often drawn from the results of correlations between drawing performance and performance on other psychological tests. Since such complex tests are sensitive to more than one kind of cerebral disorder, Warrington et al. contend that there is the possibility that the two groups of patients do badly on any pair of complex tests for different reasons. They designed a study which would eliminate these problems. The test they used consisted of four sets: 
Set I began and ended with the copying of a cube and a star. Between the two trials with the cube and star were a series of line drawings that consisted of segments or portions of the cube and star so that in effect the patient would have practiced elements of the drawings between the first and second attempts.

Set II required the patient to make two attempts each at copying the size and position of two dots placed in horizontal and diagonal positions on the stimulus models.

Set III consisted of two sets of eight geometric figures. The first or "structured" set contained internal lines which presumably would be aids in drawing the figures, while the second "unstructured" set had only outlines of the figures.

Set IV required the patient to draw free-handed, without a model to copy, a clock, house, bicycle, and face.

To establish whether groups of patients with known right or leftsided brain lesions made errors in drawings related to their neurological impairment, an independent judge evaluated a first set of drawings. The judge had neither medical nor psychological knowledge. The drawings consisted of a copied star and cube, as well as free-style drawings of a house, clock, bicycle, and face. The drawings were rated on a scale of 1 to 4 , 1 being very bad and 4 being satisfactory. No extra credit was given for good drawing ability. The performance ratings were found to form a continuum from grossly impaired to completely correct without an obvious delineation between those with and without impairment. The median rating was therefore selected to divide the groups into those having 
the greater drawing deficit (the positive group) or the lesser deficit (the negative group). The differences in overall severity of drawing disability of the left-sided and right-sided positive groups did not reach significance.

Each positive group was compared with its respective negative group for the incidence of visual, somatosensory, and motor deficits. No significant differences were found. When the two positive groups and two negative groups were compared for these deficits, both right hemisphere groups were found to have significantly higher incidence of deficits. These results demonstrate that incidence of visual, somatosensory, and motor deficits cannot be directly related to drawing ability, but instead to hemisphere differences.

Patients positive for right-sided lesions scored significantly better on the Wechsler Adult Intelligence Scale verbal subtests than did patients with left hemisphere lesions, presumably because of left hemispheric dominance for speech. On the four performance WAIS subtests, however, no significant differences were found between the left and right hemisphere groups or between groups positive for deficits.

Correlations between drawing performance and performance on the four subtests administered (Picture Completion, Picture Arrangement, Block Design, and Object Assembly) were positive for both patient groups. Drawing performance correlated highest with the object Assembly subtests, presumably because of the high "spatial loading" on Object Assembly. No significant differences in performance were found between the two patient groups on this or any of the other three subtests. The researchers raised the 
possibility that this lack of patient group differences was attributable to the complexity of the tasks and the need for a variety of skills for correct performance.

Performance characteristics were then examined to see if there were disassociations between the two groups of patients in relation to the predominant types of errors on the drawing tasks. The following results were reported.

(1) An increased number of right angles among line drawings of a cube copied by left-sided cases, not found among copies produced by right-sided cases.

(2) A tendency for the left-sided group to widen and the right-sided group to reduce the angles constituting the points of a copied star.

(3) A greater tendency to asymmetry among the drawings of right than left-sided cases.

(4) A lesser tendency on the part of left than of rightsided cases to build up complex geometrical figures systematically from their parts.

(5) A tendency for the left-sided cases to include fewer details in their freehand drawing of a house than the rightsided cases.

There was also a tendency for the left-sided cases, but not the right-sided cases, to benefit from systematic practice in copying elements of the cube.

No systematic way was found of using the freehand drawings 
of face, bicycle, and clock to discriminate between the rightand left-sided groups.

The results support the view that failure in drawing may be indicative of more than one underlying disorder. The types of errors made by patients with right hemisphere lesions suggest that these patients have difficulty in incorporating spatial information into their drawing performance, leading to disproportion and faulty articulation of parts of the drawing, while the patients with left hemisphere lesions seemed to experience difficulty in planning the drawing process, leading to simplified versions of the model.

Studies have shown that the two hemispheres make different contributions to the complex task of drawing, and that a lesion or disorder in either hemisphere can produce impaired performance (McFie, Piercy, \& Zangwill, 1950; McFie \& Piercy, 1952; Critchley, 1953; Piercy, Hécaen, \& DiAjuriagerria, 1960; Warrington, James, \& Kinsbourne, 1966; Russell, Neuringer, \& Goldstein, 1970; McFie, 1975). These studies differentiate between contributions of the hemispheres based on qualitative differences in predominant errors made by patients with known lesions.

Gazzaniga, Bogen and Sperry (1965) demonstrated, for example, that the ability to produce a three-dimensional perspective in drawing is mediated by the right hemisphere. Two patients who had had the cerebral commissures split for therapeutic purposes could copy a three-dimensional cube only with the left hand, even though drawing in general was easier to control with the right hand for both these patients. 
Lesions located in the left hemisphere create difficulties classically described as constructional apraxia, i.e., the disturbance that manifests in the formulation and execution of a drawing. Oversimplification may also be a characteristic of disturbance in this hemisphere.

In patients with right hemisphere lesions, free drawing may reveal neglect of the left half of the page or left half of elements on the page (Lezak, 1976). Inability to maintain proportions due to difficulty with spatial perception and a tendency toward overelaborateness may be present. Drawing disability tends to be more severe when lesions are located in the right hemisphere (Critchley, 1953; Milner, 1954).

The parietal lobe appears to be the critical region of the hemisphere responsible for production of drawings. However, not everyone who has sustained parietal lobe damage displays drawing difficulty (Warrington et a1., 1966).

Patients with left parietal lesions have the disability described as constructional apraxia evidenced by constriction of the response and difficulty in manipulation. Patients with right parietal lesions have spatial agnosia (McFie \& Piercy, 1952; McFie \& Zangwill, 1960; Piercy, 1964).

In the present study, the Wechsler Adult Intelligence Scale (WAIS) and the General Aptitude Test Battery (GATB) were administered with the Bicycle Drawing Test. The WAIS is a widely used test that of ten constitutes a substantial portion of the framework of a neurological examination. The Wechsler scales have been the intellectual ability tests of choice for many neuropsychologists who have incorporated them into both clinical 
and research batteries (e.g. Russell, Neuringer, \& Goldstein, 1970; Rei$\tan \&$ Davison, 1974; Smith, 1975; Lezak, 1976).

Factor analyses have shown the Block Design and Object Assembly subtests of the Wechsler Adult Intelligence Scale (WAIS) to load highly on a spatial/performance factor. Clinical studies have shown both subtests to be sensitive to parietal lobe lesions, particularly those in the right hemisphere (Fitzhugh \& Fitzhugh, 1964; McFie, 1975). This sensitivity was originally demonstrated by McFie and Piercy in 1952. They report (excluding statistics): "mean losses on Block Design are significantly greater with right. . than with left-sided.. . lesions... In each hemisphere, parietal lesions are associated with significantly greater impairment than are frontal lesions... At the same time, right parietal lesions are associated with significantly greater impairment than are left parietal lesions ..." (McFie \& Piercy, 1952, p. 304).

The GATB was included in the current study because it provided a second criterion for correlations with the Bicycle Drawing Test and because its scores were available for all participants. This test, published by the U.S. Department of Labor (1965) examines a wide range of aptitudes and abilities, the primary purpose of which is to provide information for job counseling. The thorough factoral analysis of the subtests recommends their use for both neurological research and clinical problems (Lezak, 1976)

Several subtests of the GATB measure abilities which contribute to high scores on the Bicycle Drawing Test and significant correlations between the two measures would be expected. Three-Dimensional space requires 
the participant to relate two and three-dimensional aspects to the same figure and is related to spatial aptitude. Tool Matching and Form Matching both involve perceptual accuracy and a form perception factor (Lezak, 1976).

The present study includes only men. Guilford (1967) has found that males excel in spatial ability. There are also sex differences in performance on the WAIS subtests; men do better on 5 of 11 subtests, women do better on 3 (Matarazzo, 1972). Therefore, in the interest of simplicity, women are not included in this study.

Because the performance of adults on tests of intelligence changes with age (Wechsler, 1958), age is a factor examined in this study. Five age categories were used with the same age groupings as used for the WAIS: $20-24,25-34,35-44,45-54$, and 55-64 years of age. Handedness of participants was noted for comparison purposes.

As a free drawing task, the Bicycle Drawing Test requires use of the same areas of the brain as the Block Design and Object Assembly subtests. Therefore it is hypothesized that significant correlations exist between performance on these subtests and the Bicycle Drawing Test. It is further hypothesized that significant correlations exist between the Bicycle Drawing Test and GATB subtests Three-Dimensional Space, Form Matching, and Tool Matching for reasons discussed above. While age differences and differences between right and left handed persons are examined, no directional predictions are made with regard to the age and handedness variables. 


\section{Method}

\section{Subjects}

Selected adult patients at the Workman's Compensation Disability Prevention Center, now called Callahan Center, in Wilsonville, Oregon were invited to participate in the study. These people are workers who had been injured on the job and who had not yet returned to work. Selection for participation was based on the following criteria: male, no history of neurological disorder, physically able to participate, capable of completing the GATB which requires sixth grade reading proficiency. Efforts were made to find at least 20 subjects for each of five age groups: 20-24, 25$34,35-44,45-54$, and 55-64.

Breakdown of age groups of participants finally identified for the study was:

$\begin{array}{lc}\begin{array}{l}\text { Age group } \\ \text { in years }\end{array} & \begin{array}{l}\text { Number of } \\ \text { participants }\end{array} \\ 20-24 & 21 \\ 25-34 & 46 \\ 35-44 & 37 \\ 45-54 & 27 \\ 55-64 & 10\end{array}$

Although only ten persons aged .55-64 were available to participate in the study, data from this age group were included in order to provide some information on the functioning of older men.

The population from which the participants were drawn generally consists of blue collar workers including occupational groups such as mill workers, loggers, warehouse workers, construction workers, and nurse's 
aides.

The range of school years completed was from 5 to 16 years. In the sample, $8 \%$ had eighth grade education or less, $31 \%$ had some high school training but no diploma, $47 \%$ had high school diploma or GED, and $14 \%$ had completed one to four years of college.

\section{Instruments}

Wéchsler Adult Intelligence Scale. The Wechsler Adult Intelligence Scale (WAIS) consists of a test battery of eleven different subtests. Wechsler has classified the first six as "verbal" tests and the other five as "performance" tests.

Raw scores of the subtests are usually converted to scaled score equivalents. Then the six scaled scores of the verbal section of the test are added together to form a verbal score. The five scaled scores of the performance section of the test are added together to form a performance score. Those two scores are then added together to form a full scale score. These three scores are then converted to age group normed IQ equivalents. This procedure was not used for the present study. Raw scores were converted to age group normed scaled score equivalents.

A brief description of the subtests of the WAIS follows. Normative data regarding means and standard deviations for subtests of the WAIS are presented in Appendix $A_{1}$.

The subtest Information (I) examines the range of knowledge, interest in and ability to recall facts about the world. Range of scores is 0 to 29. 
The subtest Comprehension (C) examines ability to reason, use good judgment and understand usual cultural expectations. Range of scores is 0 to 28 .

The subtest Arithmetic (A) examines the ability to concentrate and to apply logical arithmetic reasoning to the solution of problems. Range of scores is 0 to 18 .

The subtest Similarities (S) examines verbal concept formation, and ability to perceive the relations among events and organize them on an abstract conceptual level. Range of scores is 0 to 26.

The subtest Digit Span ( $D S p)$ examines the immediate auditory recall for numbers. Range of scores is 0 to 17 .

The subtest Vocabulary (V) examines potential for meaningful verbal communication. Range of scores is 0 to 80 .

The subtest Digit Symbol (DSy) examines fine motor control and coordination, accurate visual perception and discrimination. Range of scores is 0 to 90 .

The subtest Picture Completion (PC) examines visual recognition. Range of scores is 0 to 21 .

The subtest Block Design (BD) examines visual perception and ability to analyze unfamiliar, increasingly complex wholes into their parts and the organization of those parts into meaningful patterns. Range of scores is 0 to 48 .

The subtest Picture Arrangement (PA) examines understanding of interpersonal situations. Range of scores is 0 to 36 .

The subtest Object Assembly (OA) examines the ability to form a whole 
from unfamiliar segments. Range of scores is 0 to 44.

General Aptitude Test Battery. The General Aptitude Test Battery (GATB) is published by the United States Department of Labor (1970). The purpose of this test is primarily to provide information for job counseling and secondarily for educational guidance. This test is not available for commercial use but is given free of charge by state employment services and other non-profit agencies such as high schools. There are twelve subtests in this battery of which seven are multiple choice paper and pencil tests. The five other subtests involve aspects of motor speed and coordination. The manual describes the subtests in the following way (United States Department of Labor, 1970, pp. 15-17):

Part 1 - Name Comparison

This test consists of two columns of names. The examinee inspects each pair of names, one in each column, and indicates whether the names are the same or different. It measures a factor called clerical perception. The range of possible scores is from 0 to 150 with a mean of 43.715 and a standard deviation of 15.991 .

Part 2 - Computation

This test consists of a number of arithmetic exercises requiring the addition, subtraction, multiplication or division of whole numbers. It measures numerical aptitude (N). The range of scores is from 0 to 50 with a mean of 23.092 and a standard deviation of 6.725 . 
Part 3 - Three-Dimensional Space

This test consists of a series of exercises containing a stimulus figure and four drawings of three-dimensional objects. The stimulus figure is pictured as a flat piece of metal which is to be either bent or rolled or both. Lines indicate where the stimulus figure is to be bent. The examinee indicates which of the four drawings of the three-dimensional objects can be made from the stimulus figure. This test is associated with spatial aptitude (S) and has a range of 0 to 40 with a mean of 16.815 and a standard deviation of 6.523 .

Part 4 - Vocabulary

This test consists of sets of four words. The examinee indicates which two words have either the same or opposite meanings. It measures verbal aptitude (V), with a range of scores from 0 to 60 and a mean of 19.772 and a standard deviation of 10.053 .

Part 5 - Tool Matching

This test consists of a series of exercises containing a stimulus drawing and four black and white drawings of simple shop tools. The examinee indicates which of the four black and white drawings is the same as the stimulus drawing. Variations exist only in the distribution of black and white in each drawing. The test measures form perception $(P)$. The range of scores is 0 to 49 with a mean of 29.123 and a standard deviation of 6.619 . 
Part 6 - Arithmetic Reason

This test consists of a number of arithmetic problems expressed verbally and measures numerical aptitude (N). The range of scores is 0 to 25 with a mean of 11.426 and a standard deviation of 3.511 .

Part 7 - Form Matching

This test consists of two groups of variously shaped line drawings. The examinee indicates which figure in the second group is exactly the same size and shape as each figure in the first or stimulus group. This is another test of perceptual accuracy and is associated with form perception (P). Part 8 - Mark Making

This test consists of a series of squares in which the examinee is to make three pencil marks working as rapidly as possible. The marks to be made are short lines, two vertical and a third horizontal line beneath them. This test measures motor coordination (K), with a range of scores from 0 to 130 and a mean of 69.477 and a standard deviation of 10.321 .

Part 9 - Place

The equipment used for this test and for Part 10 consists of a rectangular pegboard divided into two sections, each section containing 48 holes. The upper section contains 48 cylindrical pegs. The examinee removes the pegs from the holes in the upper part of the board and inserts them into 
the corresponding holes in the lower part of the board, moving two pegs simultaneously, one in each hand. This performance is done three times with the examinee working rapidly to move as many of the pegs as possible during the time allowed for each of the three trials. The test measures manual dexterity (M), with a range of scores from 0 to 144 , a mean of 89.795 and a standard deviation of 8.615 .

Part 10 - Turn

The equipment described under Part 9 is also used for this test. For Part 10, the lower section of the board contains 48 cylindrical pegs. The examinee removes a wooden peg from a hole, turns the peg over so that the opposite end is up and then returns the peg to the hole from which it was taken, using only his preferred hand. The examinee works rapid1y to turn and replace as many of the 48 cylindrical pegs as possible during the time allowed. Three trials are given for this performance. It measures manual dexterity with a range of scores from 0 to 144 and a mean of 100.846 and a standard deviation of 9.646 .

Part 11 - Assemble

The equipment used for this test and for Part 12 consists of a small rectangular board (finger dexterity board) containing 50 holes and a supply of small metal rivets and washers. The examinee takes a small metal rivet from a hole in the upper part of the board with his preferred hand and at the same time 
removes a small metal washer from a vertical rod with the other hand; the examinee puts the washer on the rivet and inserts the assembled piece into the corresponding hole in the lower part of the board using only his preferred hand. The examinee works rapidly to remove and replace as many rivets and washers as possible during the allowed time. This test also measures finger dexterity (F) with a range of scores from 0 to 50, a mean of 29.507 and a standard deviation of 3.737 .

Part 12 - Disassemble

The equipment used for this test is the same as that described in Part 11. The examinee removes the small metal rivet of the assembly from a hole in the lower part of the board, slides the washer to the bottom of the board and puts the washer on the rod with one hand and the rivet in the corresponding hole in the upper part of the board with the other (preferred) hand. The examinee works rapidly to move and replace as many rivets and washers as possible during the time allowed. This test also measures finger dexterity (F) with a range of scores from 0 to 50 , a mean of 29.507 and a standard deviation of 3.737 .

The Bicycle Drawing Test. The Bicycle Drawing Test is a free-style drawing elicited by the presentation of paper and pencils and the instructions, "Draw a bicycle. Do the very best job you can" (Lezak, 1977). The 
test was scored by judges or raters who had developed a high level of agreement on whether the drawings met the scoring criteria devised by Muriel Lezak. The scoring criteria consists of a list of 20 items with a possible score of one point for each item. The list of items is included in Appendix $B$.

\section{Procedure}

Each subject took the GATB under standard conditions in a small group setting as a usual part of the testing procedure at the Callahan Center. Raw scores obtained on each of the 12 subtests were used for the study. (Typically, scores on subtests are combined in various ways to arrive at nine overall aptitude scores.) The use of raw scores for each subtest simplifies the task of determining which of the subtests correlate with the Bicycle Drawing Test.

The WAIS was administered and scored according to the standard procedure established by Wechsler (1955). However, in addition to the usual scoring procedure to produce Verbal IQ, Performance IQ, and Full Scale IQ, the raw scores obtained on the subtests were converted to age-normal scaled scores as suggested by McFie (1975).

Immediately following completion of the WAIS, every male patient between 20 and 64 years of age who met selection criteria was invited to participate in the study. The following invitation was extended by the psychometrist who had just completed the WAIS with the patient:

The test you have just completed is required of everyone who comes to the Center for evaluation and treatment. The follow- 
ing simple task is not a required part of the testing procedure. It is for a research project to find out how normal American men perform on this test. Whether or not you participate is entirely up to you. In keeping with federal regulations for research, your name will not be on your test. The results will be confidential and will in no way affect your diagnosis or treatment here. The task takes only a few minutes. Your participation will be appreciated. Those patients who agreed to participate were asked a series of questions selected to screen out persons with possible undiagnosed brain damage. (See Appendix C.) Regardless of the content of their answers to these questions, all participants were asked to complete the test; no one was allowed to think he had been eliminated from the study.

The administration of the Bicycle Drawing Test followed the procedure described by Muriel Lezak (1976). Three sharpened number-two pencils with good erasers and a piece of unlined $(20 \times 27 \mathrm{~cm})$ white paper were placed in front of the person with the short side of the paper near the edge of the table. The instructions were "Draw a bicycle. Do the very best job you can." To those persons who sought more information regarding type of bicycle, how large it should be, and so forth, the reply was given that the instructions simply called for the drawing of a good bicycle. The person who asked to erase or turn the paper over to begin again was given permission without special encouragement. The total time typically ranged from three to ten minutes.

In order to obtain a measure of test-retest reliability, two to five 
weeks after their original test a randomly selected sample of participants was asked to draw another bicycle. Without prior notification and at a time when each was waiting for an appointment of a different nature, the participant was asked to accompany the examiner to a nearby room. He was there asked for his cooperation in the study by drawing another bicycle. The administration of the retest was the same as for the initial test. 


\section{$\underline{\text { Results }}$}

On the 20-item Bicycle Drawing Test, total scores ranged from two to twenty, with a mean of 13.70 and a standard deviation of 3.81 . A relative frequency polygon presented in Figure 1 displays the distribution of the obtained scores. An index of skewness was computed for this distribution using the third moment and equaled -.653, indicating a negative skew.

This study was concerned mainly with reliability and validity of the Bicycle Drawing Test. Various types of reliability were examined at both the total test and individual item levels. The reliability results were used in turn to evaluate the scoring criteria for the test items. The construct validity of the Bicycle Drawing Test was evaluated by correlating it with WAIS and GATB subtests thought to measure attributes similar to those assessed by the Bicycle Drawing Test.

Reliability of the Bicycle Drawing Test.

Three types of reliability procedures were employed in the current study: interscorer reliability, test-retest reliability, and internal consistency reliability.

Interscorer Reliability. Two scorers independently scored a set of 30 bicycle drawings from the total sample of 141 . Two statistical methods were used to determine scorer reliability. First, both item and total scores from Scorer 1 were correlated with corresponding item and total scores from Scorer 2. The interscorer reliability for the total scores was .968. Correlations between the scorers' ratings for each item are 


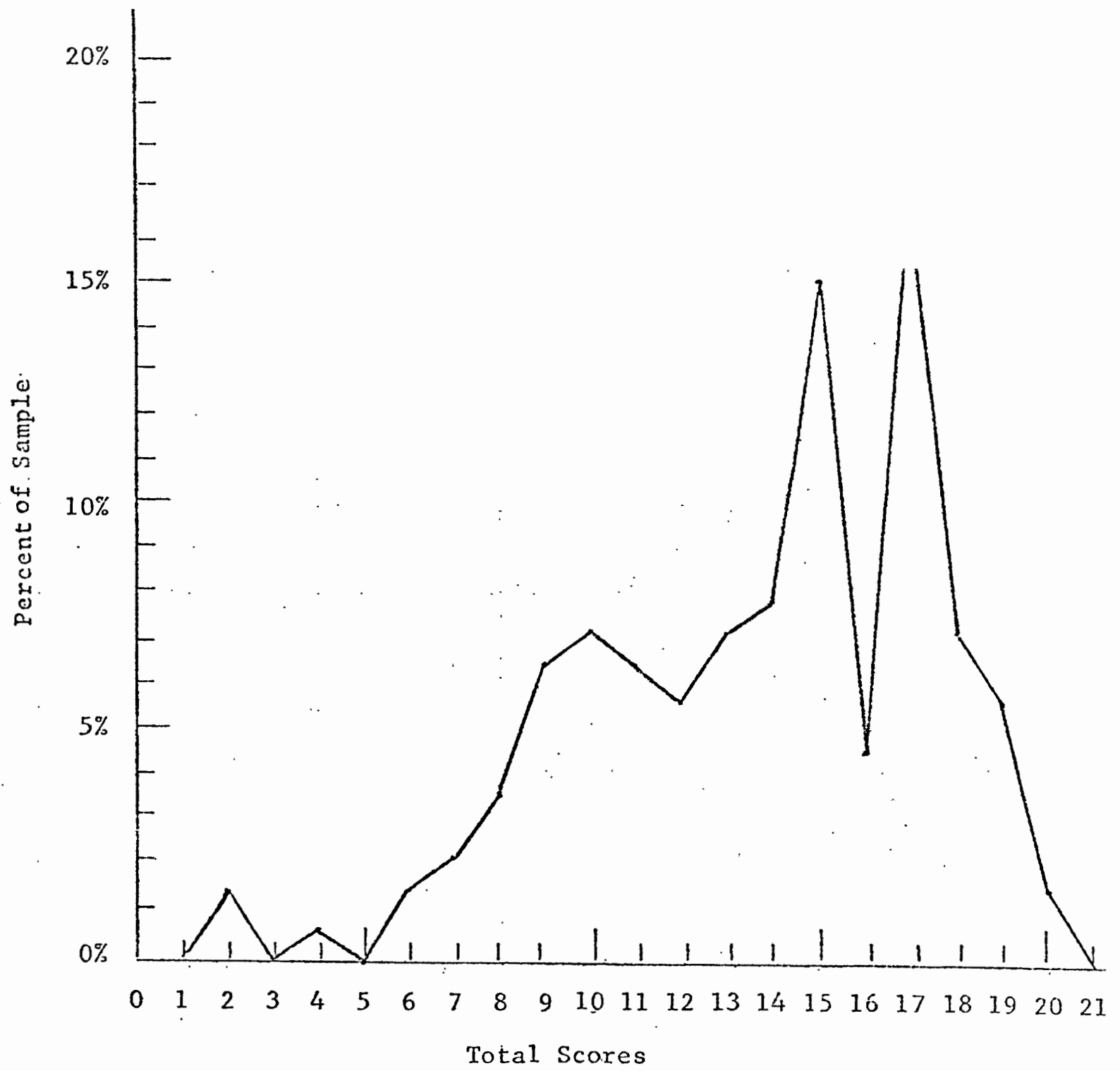

Figure 1. Relative Frequency Polygon for Bicycle Draving Test Scores. 
presented in Table 1. Items having the lowest reliabilities were 3 (.473), $14(.539), 20(.681), 10(.693), 6(.707)$, and $4(.736)$.

A second method of determining scorer reliability for each item was based on the percentage (or proportion) of bicycles for which the scorers were in complete agreement on scoring. The scorer agreement for each of the twenty items is also presented in Table 1 . The lowest rate of agreement (.867) occurred in scoring items 4, 6, 10, and 14.

Test-Retest Reliability. Test-retest reliability for total scores was determined using a Pearson correlation and was found to be only .529 . Test-retest correlations were also computed for individual items. The lowest correlations occurred for items $2(.071), 3(.172), 10(.004), 14$ $(-.083), 16(.056)$, and $19(.180)$.

Students' $\underline{t}$ tests were used to determine which item means differed significantly between Test 1 and Test 2 and to examine whether all the changes in item means were positive, indicating improvement between the first and second trials of bicycle drawing. Significant Test 1 to Test 2 change was found for eight items. Changes in six items were in a positive direction. However unexpected changes on two items (5 and 14) indicated poorer performance on Test 2 than on Test 1 . Values for the $\underline{t}$ tests for items are presented in Table 2. A $\underline{t}$ test of the difference between the means of total scores for Test 1 and Test 2 revealed significant overall improvement on the second trial $(\underline{t}(35)=-3.18, \underline{p}<.003)$.

Internal Consistency Reliability. Corrected item-total correlations and coefficient alpha were used to determine the internal consistency re1iability of the Bicycle Drawing Test. A corrected item-total correlation 
Interscorer Reliability

on Bicycle Drawing Test

\begin{tabular}{|c|c|c|}
\hline $\begin{array}{l}\text { Bicycle } \\
\text { Test Item }\end{array}$ & Pearson $\underline{r}$ & $\begin{array}{l}\text { Percentage of } \\
\text { Agreement }\end{array}$ \\
\hline 1 & undefined & 1.0 \\
\hline 2 & 1.00 & 1.0 \\
\hline 3 & .473 & .900 \\
\hline 4 & .736 & .867 \\
\hline 5 & 1.00 & 1.00 \\
\hline 6 & .707 & .867 \\
\hline 7 & undefined & 1.00 \\
\hline 8 & 1.00 & 1.00 \\
\hline 9 & .800 & .900 \\
\hline 10 & .693 & .867 \\
\hline 11 & .861 & .933 \\
\hline 12 & .932 & .967 \\
\hline 13 & .780 & .900 \\
\hline 14 & .539 & .867 \\
\hline 15 & .791 & .900 \\
\hline 16 & 1.00 & 1.00 \\
\hline 17 & .935 & .967 \\
\hline 18 & .867 & .933 \\
\hline 19 & .796 & .900 \\
\hline 20 & .681 & .933 \\
\hline
\end{tabular}

Note: If, for any item, one or both raters scored all bicycles either 1 or 0 , the Pearson correlation was undefined due to having a zero as its denominator. 
Table 2

Test-Retest Reliability

$\underline{t}$ Tests on Means of Items and Total

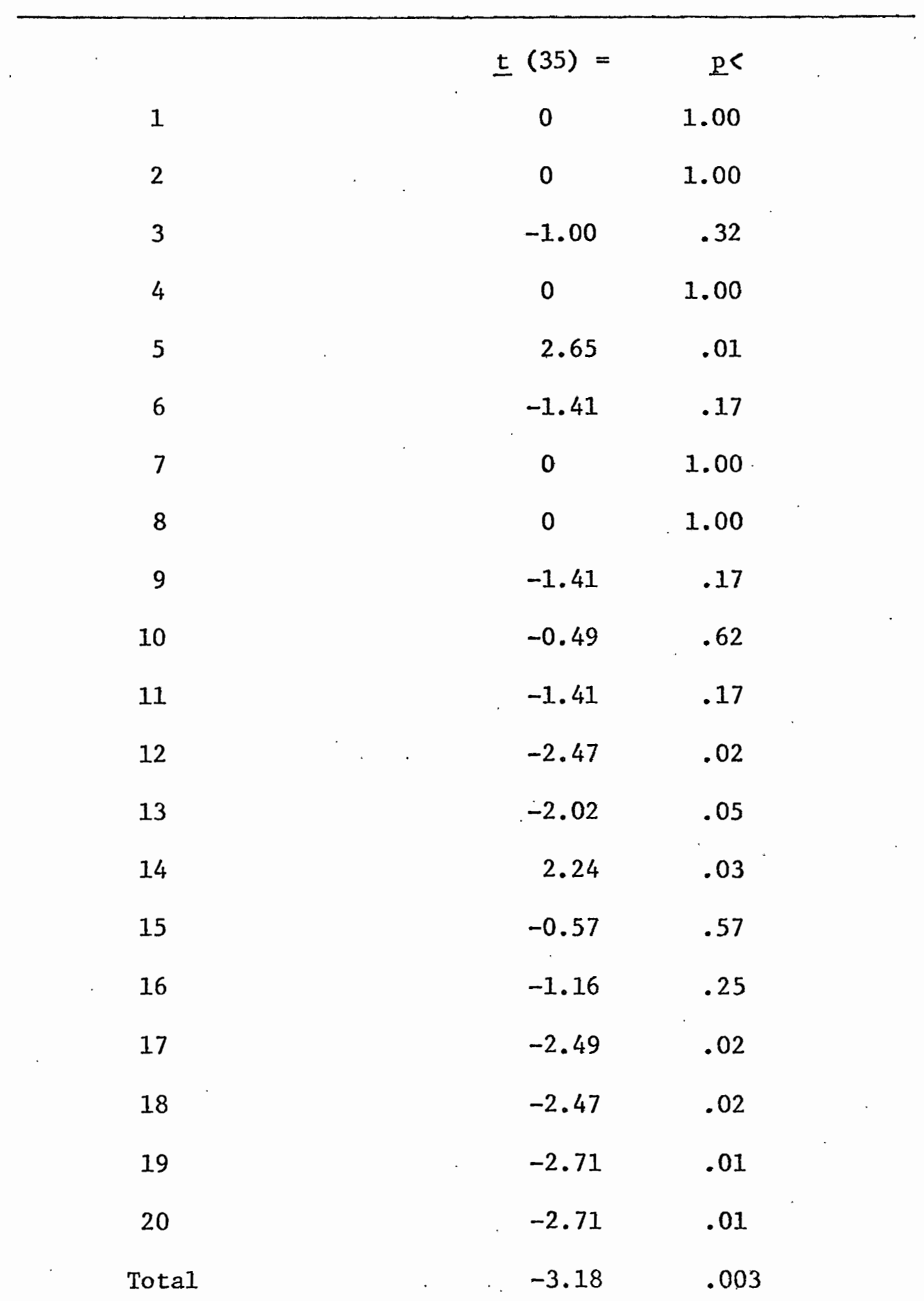


is the correlation of scores on a specific item of the scoring criteria with the total scores for all items except that the item being examined has been subtracted from the total. This gives a truer picture of how well a specific item correlates with the total because it avoids correlating the item with itself as part of the total. Items 2, 3, and 20 of the Bicycle Drawing Test scoring criteria had low correlations with the corrected total scores. Refer to Table 3 to find values for corrected item-total correlations.

The overall internal consistency of the twenty-item test was determined by coefficient alpha which equaled .80 .

Validity of the Bicycle Drawing Test.

A Pearson $\underline{r}$ was used to compute the correlation of Bicycle Drawing Test total scores with scores of WAIS and GATB subtests. For this study, an $\underline{r}$ of .317 or greater was used as a criterion for evidence of a meaningful validity coefficient. For a correlation greater than or equal to .317, it can be said that at least $10 \%$ of the variance in the subtest scores can be accounted for by the variance in the Bicycle Drawing Test scores. Correlations of five of the eleven WAIS subtests, including Block Design, Object Assembly, Picture Completion, Similarities, and Information, with the . Bicycle Drawing Test met this .317 criterion. Two GATB subtests met this criterion, Three-Dimensional Space and Form Matching. Although Vocabulary and Tool Matching were significantly correlated with the Bicycle Drawing Test, their correlations did not exceed the .317 cutoff. The values of the correlations of WAIS and GATB subtests with the Bicycle Drawing Test 
Table 3

Item Difficulty and Corrected Item - Total Correlations

for the 20 Bicycle Drawing Test Items

\begin{tabular}{|c|c|c|c|c|}
\hline $\begin{array}{l}\text { Bicycle } \\
\text { Test Item }\end{array}$ & $\begin{array}{c}\text { Item } \\
\text { Difficulty }\end{array}$ & $\begin{array}{l}\text { Corre } \\
\text { Total }\end{array}$ & $\begin{array}{l}\text { cted Item- } \\
\text { Correlation }\end{array}$ & \\
\hline 1 & .993 & & .242 & \\
\hline 2 & .837 & & .182 & \\
\hline 3 & .844 & & .190 & \\
\hline 4 & .560 & & .325 & \\
\hline 5 & .858 & & .403 & \\
\hline 6 & .695 & & .500 & \\
\hline 7 & .978 & & .334 & \\
\hline 8 & .972 & , & .253 & \\
\hline 9 & .496 & & .357 & \\
\hline 10 & .667 & & .326 & \\
\hline 11 & .624 & & .341 & \\
\hline 12 & .652 & & .558 & \\
\hline 13 & .745 & & .596 & \\
\hline 14 & . 910 & & .410 & \\
\hline 15 & .688 & & .540 & \\
\hline 16 & .780 & & .477 & \\
\hline 17 & .496 & & .613 & \\
\hline 18 & .433 & & .353 & \\
\hline 19 & .319 & & .282 & \\
\hline 20 & .206 & & .154 & \\
\hline
\end{tabular}


are presented in Tables 4 and 5.

Other Evaluations of the Bicycle Drawing Test.

A $t$ test was used to see whether handedness has a significant effect on performance on the Bicycle Drawing Test. The mean total score for the 123 right-handed participants in the study was 13.78 , with a standard deviation of 3.79. The mean total score for the 17 left-handed participants was 12.94, with a standard deviation of 3.99 . The difference in these means was not significant $(\underline{\mathrm{t}}(138)=.85, \underline{\mathrm{p}}$ <.397).

Effect of age on Bicycle Drawing Test performance was examined with a one-way analysis of variance. Drawing ability, as measured by this test, was not different for the five age categories $(\underline{F}(4,136)=.771, \underline{p}<.546)$. Means and standard deviations of total scores on the Bicycle Drawing Test for the five age categories are presented in Table 6. A table of means and standard deviations of performance on WAIS subtests by age groups is presented in Appendix $A_{2}$ for comparison with the normative data table of means in Appendix $A_{1}$. 
Table 4

Correlation of Bicycle Drawing Test

with WAIS Subtests

\begin{tabular}{lcc}
\hline \multicolumn{1}{c}{ WAIS Subtest } & \multicolumn{1}{c}{ Correlation } \\
\hline & .505 & $\underline{\mathrm{r}}$ \\
Block Design & .400 & .001 \\
Object Assembly & .379 & .001 \\
Picture Completion & .345 & .001 \\
Similarities & .329 & .001 \\
Information & .295 & .001 \\
Arithmetic & .290 & .001 \\
Vocabulary & .249 & .001 \\
Picture Arrangement & .248 & .001 \\
Comprehension & .155 & .033 \\
Digit Symbol & .131 & .062 \\
Digit Span & &
\end{tabular}


Table 5

Correlation of Bicycle Drawing Test

with GATB Subtests

\begin{tabular}{|c|c|c|}
\hline GATB Subtest & \multicolumn{2}{|c|}{ Correlation } \\
\hline & $\underline{\mathbf{r}}$ & $P<$ \\
\hline Three Dimensional Space & .488 & .001 \\
\hline Form Matching & .363 & .001 \\
\hline Vocabulary & .293 & .001 \\
\hline Tool Matching & .260 & .001 \\
\hline Arithmetic Reason & .174 & .020 \\
\hline Assemble & .154 & .035 \\
\hline Name Comparison & .123 & .074 \\
\hline Computation & .117 & .084 \\
\hline Disassemble & .097 & .126 \\
\hline Turn & .092 & .140 \\
\hline Mark Making & .079 & .178 \\
\hline Place & .035 & .341 \\
\hline
\end{tabular}


Table 6

Means and Standard Deviations

of Bicycle Drawing Test Total Scores

\begin{tabular}{llc}
\hline Age Group & Mean & Standard Deviation \\
\hline $20-24$ & 13.952 & 4.031 \\
$25-34$ & 13.783 & 3.552 \\
$35-44$ & 14.216 & 3.630 \\
$45-54$ & 12.593 & 3.651 \\
$55-64$ & 13.900 & 5.507
\end{tabular}




\section{Discussion}

The validity of the Bicycle Drawing Test gains support from the significant correlations that are found between performance on this test and certain subtests of the WAIS and GATB. Both hypotheses regarding Bicycle correlations with WAIS and GATB subtests were empirically supported.

The WAIS subtests that most strongly correlated with the Bicycle Drawing Test were Block Design and Object Assembly. The drawing task and these two subtests require visual perception and the fitting together of parts to make meaningful patterns or objects. Other studies have found these two subtests to have the highest spatial loading and to use the same areas of the brain required for drawing tasks.

Three other WAIS subtests not hypothesized to correlate highly with the Bicycle Drawing Test did, in fact, correlate with it beyond our .317 criterion. These subtests were Picture Completion, Similarities, and Information. Although it had not been predicted, the high correlation between the Bicycle Drawing Test and Picture Completion should not be surprising. Memory for design is a function of the right parietal and temporal lobes. This, together with attention to details of the environment, plays a role in accurate execution of the free-drawing task as well as the Picture Completion subtest. The subtest Similarities requires concept formation and the ability to perceive and organize relationships on an abstract level. The high correlation found here might be explained by the fact that as a free-drawing task rather than a copying task, the Bicycle Drawing Test requires the formation of a perceptual construct, necessitating an abstract- 
ing ability. The subtest Information tests interest in and ability to recall facts about the world. The correlation found here may indicate that the person who performs well on the Bicycle Drawing Test is one who is interested in and takes notice of objects in his environment.

The high correlations between performance on the Bicycle Drawing Test and the GATB subtests Three-Dimensional Space and Form Matching had been predicted. Tool Matching did not correlate as highly as expected. Although Tool Matching is thought to load on the same factors as Form Matching, there are differences in the tasks involved. Tool Matching requires the matching of a stimulus figure with one of four test figures which are nearly identical. The only differences in the figures are in the distribution of black and white. Form Matching requires the matching of size and shape of the line-drawing stimulus figure with one of four test group figures. The skills necessary for speedy performance on Form Matching more closely match those necessary for the bicycle drawing task than do skills necessary for Tool Matching.

Interscorer reliability was very high on the Bicycle Drawing Test. Although the raters had achieved a high level of agreement on the scoring of items, they found that some items were more difficult to score than other items. Scoring for those items was more difficult because they required the most subjective judgment. Most of those were the same items found to have least agreement on interscorer reliability. Those items were 3, 4, 10, 13, 14, 15, 18, 19, and 20. For those items specific definitions and/or examples regarding what are scorable responses would be useful. Such definitions and examples would be especially helpful in improving the stan- 
dardization of scoring procedures. For example, item 15 is "supporting bar from front to pedals." That statement does not specify whether the presence of the bar in approximate position earns one point or whether the bar must be properly attached to the front vertical bar and to appropriate place near the pedal mechanism to earn the point.

Test-retest reliability is fairly low. Because the Bicycle Drawing Test has been found to be valid and reliable in other ways, it could probably best be said that this is a good test of free-drawing ability for a one-trial application. For no explainable reason, on two items performance on the second trial was poorer than on the first trial. On six items there was significant improvement on the second trial. This may be due to thinking about or taking notice of bicycles in the interim between trials. On the other hand, the items that had significant change in either direction were in most cases those same items requiring the most subjective judgment by the raters. It would be interesting to see whether more clearly defining the scoring criteria would affect the test-retest reliability and in which direction any effect would be.

Another variable which was not controlled for in this study and might have a bearing on the results of the test-retest reliability analysis is experimenter effect. Four psychometrists administered the Bicycle Drawing Test after the completion of the WAIS for Test 1 . All retests were administered by one of the original four psychometrists. One participant had been given Test 1 by a young male psychometrist. When the retest was given, he made an obvious attempt to be helpful and impress the female psychometrist. His second drawing contained many of the same obvious distortions 
and omissions, but also contained more extraneous details, such as reflectors and filled-in areas.

Two other variables might have affected the test-retest reliability results. Test 1 was taken after completing the entire WAIS, which usual1y takes a minimum of an hour. Some participants had been in testing situations for one half to one full day prior to taking the WAIS. Fatigue and lack of enthusiasm may have had greater effect on the effort expended for the first test. The second trial of bicycle drawing occurred at a time when the participants had been engaged in activities other than test activities for two or more weeks. Test 2 was presented as an isolated task rather than after other testing.

It would be interesting to control for some of the variables which existed in the present study to see whether the test-retest reliability would be improved. This could be done by having the same experimenter administer both trials to people who had not been engaged in testing just prior to either trial.

The item-total correlations and coefficient alpha reflect a test that has moderate to moderately high internal consistency. This indicates that the items are testing a fairly homogeneous attribute. Scores on three of the items, items 2, 3, and 20, were not as highly correlated with the total score as would be desired. There are two reasons that may account for this result. An uneven split, almost all responses on an item being correct or wrong, may àccount for the low correlation. The other possibility is that raters may have scored the items differently. Performance on the Bicycle Drawing Test could not be predicted by 
whether the participant was left or right handed. Neither could drawing ability be predicted by knowledge of the person's age. The variables of handedness and age were found to have no effect on the test scores.

The Bicycle Drawing. Test is only appropriate to use as part of a battery of neuropsychological tests. Since normal people vary greatly in performance on this test, conclusions should not be drawn about the presence of organicity based on abilities displayed by performance on this one test.

The Bicycle Drawing Test seems to be culture-fair because bicycles are found in most cultures at all economic levels. The test does not require use of language except in the simple instructions which can be translated. The actual drawing is complex enough to require the use of circles, straight lines, angles, curves, and diagonals while maintaining proper size relationships and overall proportions.

The scoring criteria deserve some further consideration. Item 18, "fenders", created many problems. After data collection for this study was begun, one intelligent young participant commented as he was completing his drawing that he had not put fenders on the bicycle because it was a racing bike. Investigation confirmed this man's belief; racing bicycles do not have fenders. In a consultation with Muriel Lezak, the scoring on item 18 became "fenders - no fenders on racing bicycle = 1 point; fenders on any other bicycle = 1 point." It then was the task of the rater to judge whether a participant was attempting to draw a racing bicycle or whether he had forgotten the fenders. This problem could be avoided by substituting "tires," often forgotten and certainly less debatable, for 
"fenders."

On some items of the test, such as previously mentioned item 15 "supporting bar from front to pedals," one point might be given for the presence of the bar, another for its proper placement.

An interesting idea for a future study would be to test a new scoring system. The scoring criteria could consist of 2 sets: A for those items more likely indicative of left hemisphere deficit, and B for those items more likely indicative of right hemisphere deficit.

In summary, the major purpose of this study was to examine the reliability and validity of the Bicycle Drawing Test. Correlations with subtests of the WAIS and GATB offer evidence for the validity of the test. Interscorer reliability and internal consistency reliability meet standards for tests of this sort.

Test-retest reliability is not as high as desired. Possible reasons for this have been offered and suggestions have been made for future examination of variables.

Handedness and age were examined as variables. Neither was found to have a significant effect on performance on this test.

This study presents some normative data about the Bicycle Drawing Test. The data indicate that this test has an appropriate place in a battery of neuropsychological tests. Further research should focus on the various deficits of neurological functioning that are tapped by the Bicycle Drawing Test. 


\section{References}

Critchley, M. The parietal lobes. Baltimore: Williams \& Wilkens, 1953.

Fitzhugh, L.C. \& Fitzhugh, K.B. Relationships between WechslerBellevue Form I and WAIS performances of subjects with longstanding cerebral dysfunction. Perceptual and Motor Skills, 1964, 19, 539-543.

Gazzaniga, M.S., Bogen, J.E., \& Sperry, R.W. Observations on visual perception after disconnection of the cerebral hemispheres in man. Brain, 1965, 88, 221-236.

Guilford, J.P. The nature of human intelligence. New York: McGrawHill, 1967.

Lezak, M.D. Neuropsychological assessment. New York: Oxford University Press, 1976.

Lezak, M.D. Personal communication, March 5, 1977.

Matarazzo, J.D. Wechsler's measurement and appraisal of adult intelligence (5th ed.). Baltimore: Williams \& Wilkins, 1972. McFie, J. Assessment of organic intellectual impairment. New York: Academic Press, 1975.

McFie, J. \& Piercy, M.F. Intellectual impairment with localized cerebral lesions. Brain, 1952, 5, 292-311.

McFie, J., Piercy, M.F., \& Zangwi1l, O.C. Visual-spatial agnosia associated with lesions of the right cerebral hemisphere. Brain, 1950, 73, 167-190. 
McFie, J. \& Zangwill, 0.L: Visual-constructive disabilities associated with lesions of left cerebral hemispheres. 'Brain, 1960, $\underline{83}, 243-260$.

Milner, B., Intellectual functioning of the temporal lobes. Psychological Bulletin, 1954, 51, 42-62.

Piaget, J. The child's conception of physical causality, trans. New York: Harcourt, Brace, 1930.

Piercy, M., The effects of cerebral lesions on intellectual functions: a review of current research trends. British Journal of Psychiatry, 1964, 110, 310-352.

Piercy, M.D., Hécaen, H., \& DiAjuriagerria, J. Constructional apraxia associated with unilateral cerebral lesions: left and right sides compared. Brain, 1960, 83, 225-242.

Reitan, R.M. \& Davidson, L.A. Clinica1 neuropsychology : Current status and application. New York: Winston/Wiley, 1974.

Russell I, E.W., Neuringer, C., \& Goldstein, G. Assessment of brain damage. Neuropsychological key approach. New York: Wiley Interscience, 1970.

Sharma, T.R. Measuring intelligence through bicycle drawings. Indian Educational Review, 1972, $\underline{7}, 1-30$. (abstract)

Slattery, P.F. Personal communication, September 8, 1980. General Research Corporation. McLean, Virginia.

Smith, A. Neuropsychological testing in neurological disorders. Advances in Neurology, W.J. Friedlander (Ed.), New York: Review Press, 1975, $\underline{7}$. 
Taylor, E.M. Psychological appraisal of children with cerebral defects: Cambridge, Mass.: Harvard University Press, 1959. United States Department of Labor, Manpower Administration. Manual for the USES general aptitude test battery, Section III. U.S. Government Printing Office, Washington, D.C., 1970.

Warrington, E.K., James, M., \& Kinsbourne, M. Drawing disability in relation to laterality of cerebral lesion. Brain, 1966, 89, 53-82.

Wechsler, D. The measurement and appraisal of adult intelligence (4th ed.). Baltimore: Williams \&. Wilkins, 1958.

Wechsler, D. Wechsler Adult InteIligence Scale. Manual. New York: Psychological Corporation, 1955. 
Appendix $A_{1}$

TABLE OF NORMATIVE DATA FOR SUBTESTS OF THE WAIS

$$
\text { Age Groups: } \quad 20-24 \quad 25-34 \quad 35-44 \quad 45-54 \quad 55-64
$$

Subtests:

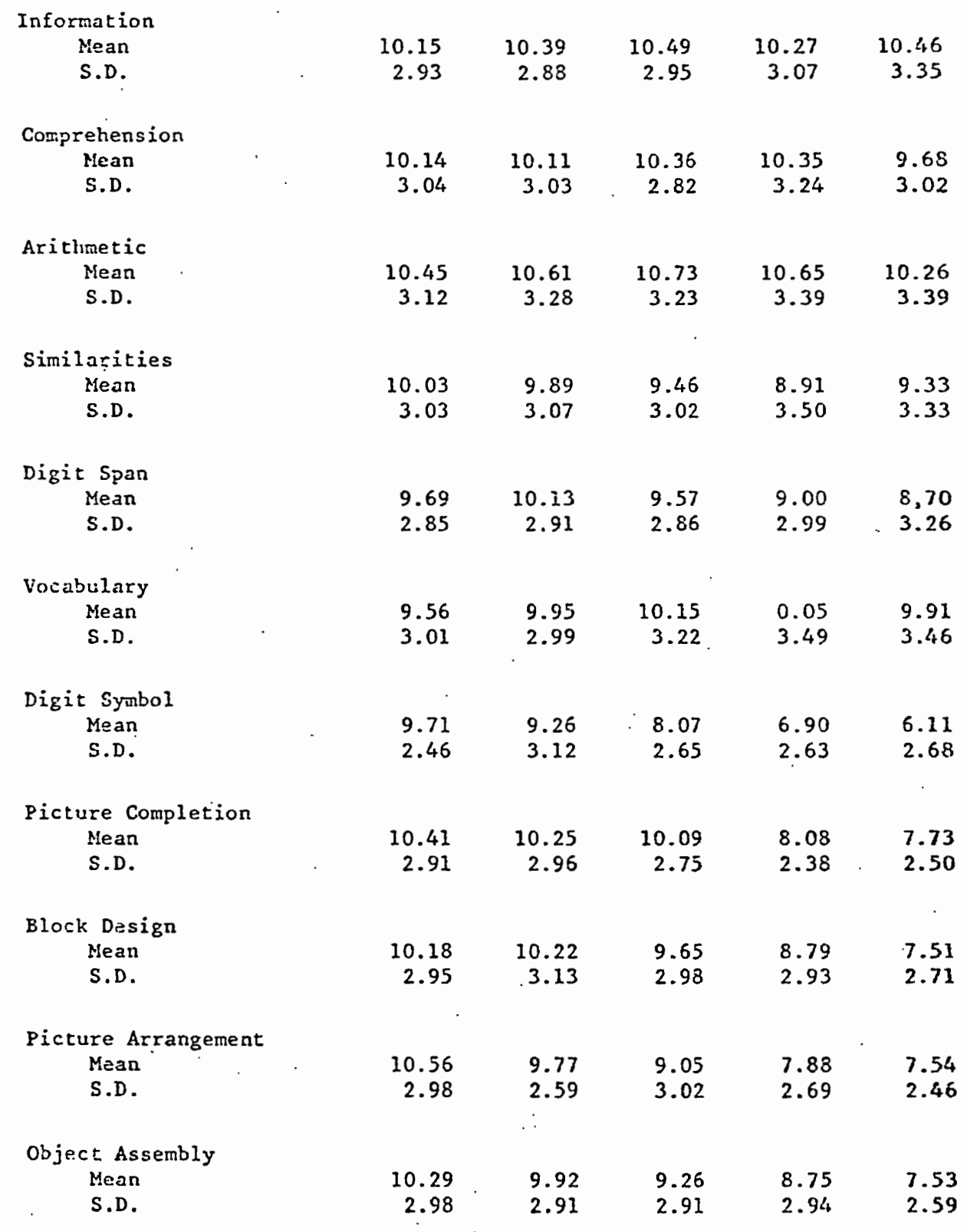




$$
\text { Appendix } \mathrm{A}_{2}
$$

MEANS AND STANDARD DEVIATIONS FOR SUBTESTS OF THE WAIS FOR THE STUDY SAPIE

$$
\text { Age Croups: } \quad 20-24 \quad 25-34 \quad 35-44 \quad 45-54 \quad 55-54
$$

\section{Subtests:}

Information

Mean

S.D.

9.67

9.69

9.51

10.59

I1. 30

1.91

2.26

2.35

1.93

1.42

Comprehension

Mean

S.D.

Arithmetic

Mean

S.D.

Similarities

Mean

S.D.

Digit Span

Mean

S.D.

Vocabulary

Mean

S.D.

Digit Symbol

Mean

S.D.

Picture Completion

Mean

S.D.

Block Design

Mean

S.D.

Picture Arrangement

Mean

S.D.

Object Assembly

- Mean

S.D.
10.60

2.66

10.76

2.43

11.30

2.03

12.00

2.36

10.71

2.12

9.93

2.55

9.35

10.81

1.73

10.90

2.56

1.45

$\begin{array}{rrrrr}11.05 & 10.76 & 10.81 & 11.04 & 10.10 \\ 2.22 & 2.87 & 2.46 & 1.85 & 1.66\end{array}$

9.76

8.80

9.00

2.80

9.56

2.28

9.80

2.70

10.14

9.90

9.76

10.22

1.87

11.30

1.85

2.23

1.42

$\begin{array}{rrrrr}10.76 & 10.09 & 10.03 & 10.96 & 11.30 \\ 2.76 & 1.64 & 2.25 & 1.74 & 1.49\end{array}$

$\begin{array}{rrrrr}11.19 & 10.80 & 11.08 & 11.93 & 13.60 \\ 1.54 & 2.16 & 2.37 & 2.11 & 1.78\end{array}$

$\begin{array}{rrrrr}11.57 & 10.69 & 10.51 & 10.74 & 12.50 \\ 2.06 & 2.39 & 2.57 & 2.75 & 3.60\end{array}$

$\begin{array}{rrrrr}10.14 & 10.00 & 10.46 & 11.59 & 11.90 \\ 1.82 & 2.33 & 2.14 & 1.78 & 3.07\end{array}$

$\begin{array}{rrrrr}10.14 & 10.60 & 10.68 & 10.85 & 13.00 \\ 2.24 & 2.88 & 2.98 & 2.32 & 3.59\end{array}$

NOTE: These scores are age-scaled scores, unlike the normative data for subtests in Table $A_{1}$ 
Appendix B

Scoring: Bicycle Drawing Test

Score one point for each of the following:

1. two wheels

2. spokes on wheels

3. wheels approximately the same size (no greater than $2 / 3$ difference)

4. wheel size in proportion to bicycle

5. front wheel shaft connected to handle bars

6. rear wheel shaft connected to seat or seat shaft

7. handle bars

8. seat

9. seat shaft connected to pedals

10. seat in workable relation to pedals (not too far ahead or behind)

11. pedals (2)

12. pedals properly placed relative to turning mechanism or gears

13. gears indicated (chain wheel and sprocket)

14. top supporting bar properly placed

15. supporting bar from front to pedals

16. drive chain

17. drive chain properly attached

18. fenders (no fenders on racing bike; 2 fenders on all others)

19. lines properly connected

20. no transparencies

Maximum possible score: 20 points 
Appendix C

Bicycle Drawing Test Questionnaire

Circle the correct answers. Explain ALL yes responses.

Have you had

A. a head injury? no yes, explain

B. a high fever? no yes, explain

C. spells of any kind? no yes, explain

D. periods when you lost consciousness? no yes, explain

E. periods when you lost control of speech? no yes, explain

F. periods when you lost control of any limb? no yes, explain

Are you naturally left or right handed?

Has anyone ever tried to change your handedness?

Age: $\quad$ Education: 